\title{
Evolution de morphologie de la baie de Coolangatta (Gold Coast, Australie) : impact des rechargements de plage et du système permanent de bypass de sédiment
}

\author{
Bruno Castelle ${ }^{1}$, Ian L. Turner ${ }^{2}$, Rodger B. Tomlinson ${ }^{3}$, Xavier Bertin ${ }^{4}$ \\ ${ }^{1}$ CNRS UMR EPOC-5805, Université Bordeaux I, \\ Avenue des Facultés, 33405 Talence Cedex, France \\ b.castelle@epoc.u-bordeaux1.fr \\ ${ }^{2}$ Water Research Laboratory, School of Civil and Environmental Engineering, \\ University of New South Wales, NSW 2093, Australie \\ ian.turner@unsw.edu.au \\ ${ }^{3}$ Griffith Centre for Coastal Management, Griffith University, PMB 50 Gold \\ Coast Mail Centre, Queensland, 9726, Australie \\ r.tomlinson@griffith.edu.au \\ ${ }^{4}$ National Civil Engineering Laboratory, Estuaries and Coastal Zones Division, \\ 1700-066, Lisbon, Portugal \\ xbertin@lnec.pt
}

\section{Résumé :}

Afin de restaurer les plages de la baie de Coolangatta (Gold Coast, Australie), un nombre important de rechargements a été réalisé ces 30 dernières années avec, en particulier, la mise en place d'un système de bypass permanent depuis 2001. Les résultats de cette étude montrent que la baie de Coolangatta s'est engraissée très rapidement depuis 2001. L'accrétion et l'avancée des plages atteignent respectivement $6 \mathrm{~m}$ et $200 \mathrm{~m}$ par endroits entre 1995 et 2005. Le système de bypass a l'impact le plus significatif sur cette forte accrétion. Ces travaux de rechargement ont délivré avec succès de grandes quantités de sédiments pour restaurer les plages de la baie, même s'ils ont été jusqu'à présent fortement controversés par la communauté locale.

\begin{abstract}
:
To achieve the objective of restoring and maintaining beach amenity, significant nourishment works have been undertaken in Coolangatta Bay (Gold Coast, Australia) over the past 30 years, with particularly the implementation of a permanent sand bypass system in 2001. Results show that Bay has infilled at an
\end{abstract}

DOI: 10.5150/jngcgc.2008.020-C $\quad$ (disponible en ligne - http://www.paralia.fr - available online) 
amazing speed since 2001. Sedimentation reaches up to $6 \mathrm{~m}$ in some area between 1995 and 2005. Beach width has increased by $200 \mathrm{~m}$ in some areas. The sand bypassing system has the most significant impact on Coolangatta Bay morphology. The engineering components of the nourishment works are a real success, although they have been up to now controversial from many community perspectives.

\section{Mots-clés :}

Bypass - Rechargements - Imagerie vidéo - Modélisation - Dérive littorale

\section{$1 \quad$ Introduction}

Traditionnellement, les côtes en érosion sont protégées par des structures en dure telles que des épis, des brise-lames ou des jetées (CHARLIER et al., 2005). Alors que ce type de structures fournit une protection efficace localement, elles ont tendance à transférer le problème d'un site à l'autre. Des avancées majeures ont été réalisées ces 30 dernières années au niveau des techniques de rechargement de plage. D'une manière générale, le rechargement de plage consiste à placer du sédiment provenant d'une source extérieure (réserve au large, embouchure tidale, etc.) le long d'une côte en érosion et ainsi favoriser l'avancée du trait de côte pour protéger le littoral contre les épisodes de tempête, fournir des plages suffisamment larges pour les touristes ou encore protéger ou restaurer des habitats naturels. Les rechargements de plage sont désormais largement utilisés partout dans le monde (HAMM et al., 2002 ; HANSON et al., 2002) avec des efficacités et des durabilités très variables. Ce papier présente les résultats préliminaires d'un large projet innovant de protection côtière associant rechargements de plage et la mise en place d'un système de bypass permanent de sédiment sur une baie anthropisée.

La baie de Coolangatta est située à la bordure des états du New South Wales et du Queensland (Figure 1). L'érosion des plages de cette baie est devenue problématique après l'extension des digues de la Tweed River réalisée au début des années 60 afin d'améliorer les conditions de navigation de l'embouchure. Ces digues ont constitué un piège pour la dérive littorale qui était la source principale de sédiment vers la baie. Au début des années 90, et ce malgré les nombreux rechargements de plage et de dragage, les plages de la baie étaient d'une part complètement érodées, et d'autre part tout le sable accumulé en amont était à nouveau disponible pour combler l'embouchure, rendant les conditions de navigation catastrophiques. Le Tweed River Entrance Sand Bypassing Project (TRESBP) a alors été mis en œuvre en 1995 afin de proposer une solution durable pour à la fois recréer les plages de la baie de Coolangatta et assurer de bonnes 
conditions de navigation. Dans le cadre de ce projet, un nombre important de rechargements utilisant le sable dragué dans l'embouchure de la Tweed River, et la mise en place d'un système de bypass de sédiment en 2001, ont été réalisés. Ce papier se focalise sur la période 1995-2005 pendant laquelle la morphologie de la baie a considérablement évolué.

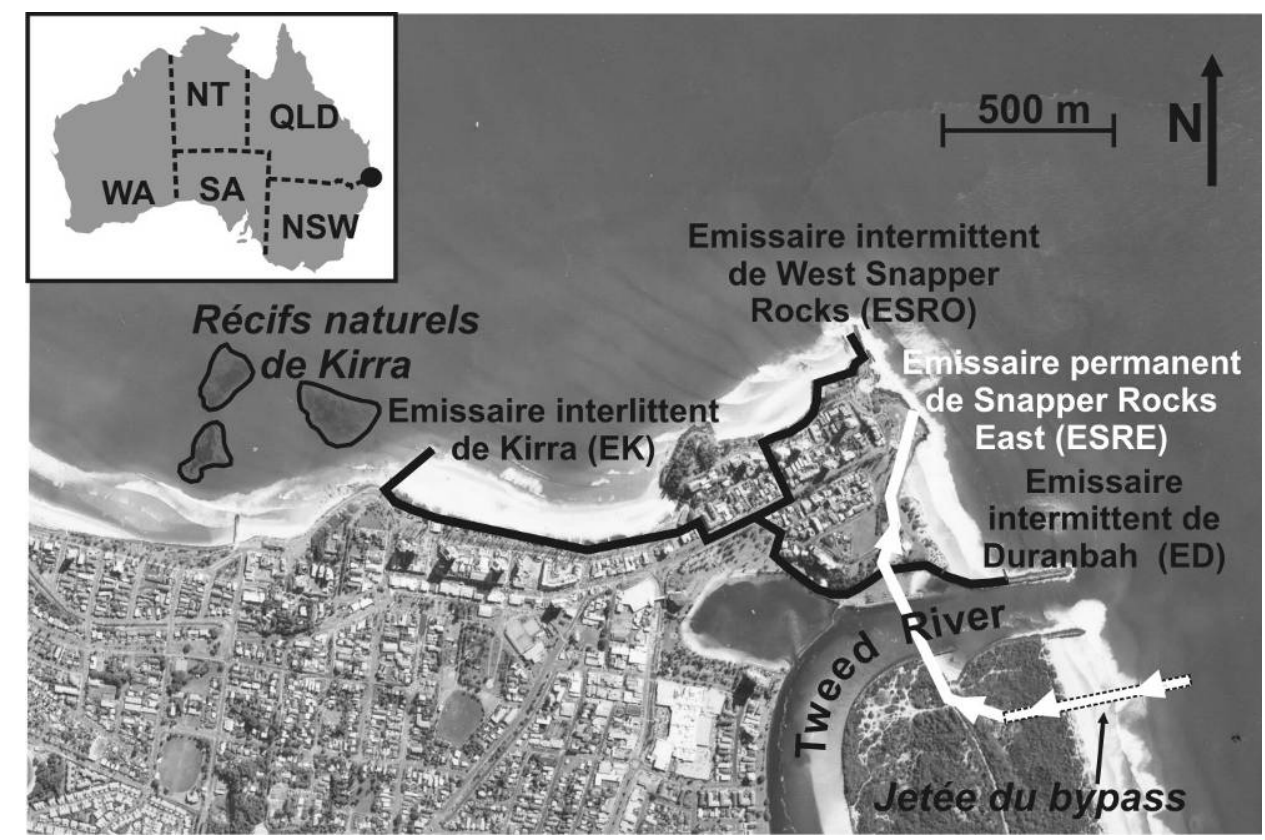

Figure 1. Localisation et configuration de la baie de Coolangatta, avec superposée la schématisation du système de bypass

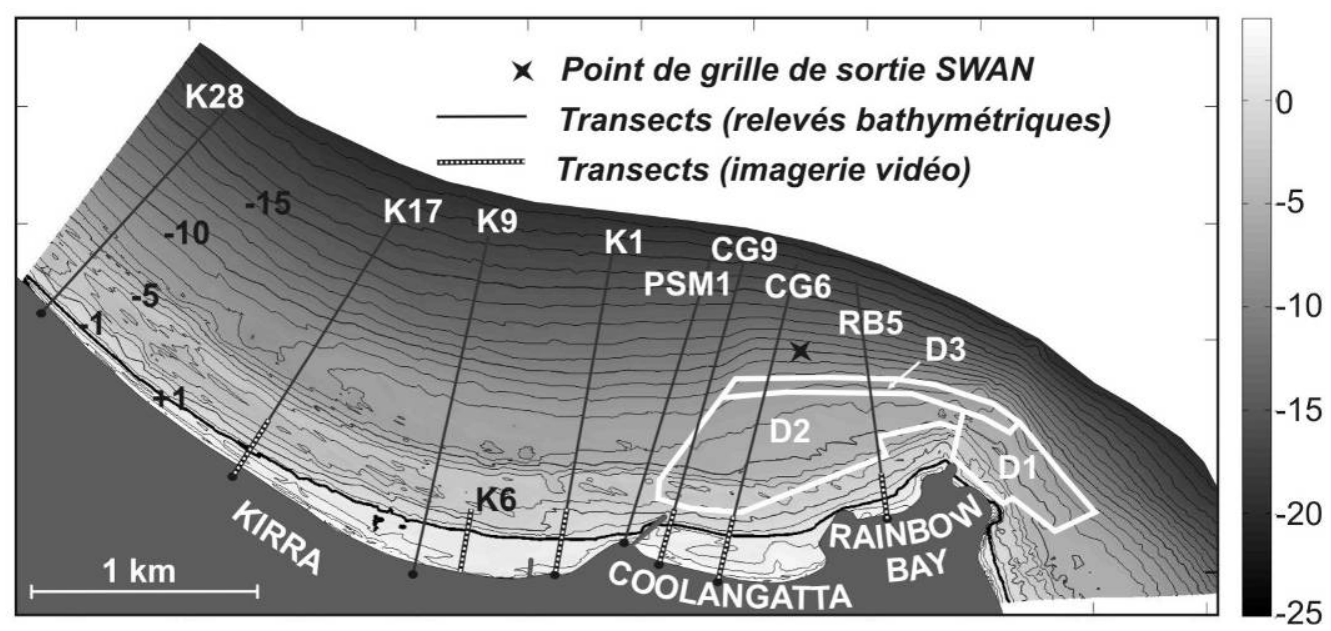

Figure 2. Morphologie de la baie de Coolangatta en 1997 avec localisation des profils de référence de relevés bathymétrique, d'imagerie vidéo, et indication des zones de dépôt (D1, D2 et D3) du sable dragué dans l'embouchure 


\section{Zone d'étude}

La baie de Coolangatta (Fig. 1) couvre environ $6 \mathrm{~km}$ de linéaire côtier et comprend 3 baies de plus petite échelle (Fig. 2) : Rainbow Bay, Coolangatta Beach et Kirra Beach. La marée est de type micro-mésotidal, semi-diurne, avec un marnage compris entre $0.2 \mathrm{~m}$ et $2 \mathrm{~m}$. Ces plages sont exposées aux houles énergétiques d'E à $\mathrm{S}-\mathrm{SE}$. Un régime de houle de $\mathrm{S}$ à $\mathrm{SE}$ est généré toute l'année, préférentiellement en hiver, qui est responsable de la composante principale de la dérive littorale dirigée vers le nord estimée à environ $500000 \mathrm{~m}^{3} /$ an (TURNER et $a l$., 2006). Le sédiment est constitué de sable fin $\left(\mathrm{d}_{50} \approx 200 \mu \mathrm{m}\right)$. La quantité de sable charrié par la Tweed River est largement négligeable par rapport à la dérive littorale.

\section{$3 \quad$ Matériels et méthodes}

\subsection{Relevés bathymétriques}

Les relevés bathymétriques réalisés par différentes organisations depuis 1987 ont été regroupés et uniformisés dans le cadre de cette étude. Huit profils de référence ont été choisis pour leur représentativité de la morphologie de la baie et sont présentés sur la Figure 2. A partir de ces profils ont été calculées les positions du trait de côte. Il existe de multiples définitions de la position du trait de côte dans la littérature (BOAK \& TURNER, 2005). Dans cette étude nous avons défini la position du trait de côte comme l'intersection du profil de plage avec le niveau moyen des mers au niveau de la Gold Coast.

\subsection{Modélisation numérique}

Le modèle de vagues SWAN (BOOIJ et al., 1999) a été couplé au modèle globale WW3 (TOLMAN, 1991). Cette stratégie de modélisation a déjà été testée avec succès sur la Gold Coast (BROWNE et al., 2006 ; 2007).

Une grille curvilinéaire plus raffinée (taille des mailles de l'ordre de $5 \mathrm{~m}$ ) a été emboitée sur la plage immédiatement au sud de l'embouchure de la Tweed River afin d'extraire les conditions de houle le long d'un profil. Les conditions de houle le long de ce profil ont ensuite été utilisées pour forcer un modèle empirique de transport sédimentaire (KACZMAREK et al., 2005) en suivant la stratégie décrite dans BERTIN et al. (2008) afin de calculer le taux de transport sédimentaire naturel «potentiel» (en l'absence des digues) pouvant entrer dans la baie de Coolangatta. La différence entre ces taux et les quantités de sable pompé et déposé dans le cadre du TRESBP (disponibles sur le site www.tweedsandbypass.nsw.gov.au/ et résumées dans les Tableaux 1 et 2) ont ainsi permis d'estimer les quantités de sable surpompé. 
Tableau 1. Quantités de sable dragué $\left(\mathrm{m}^{3}\right)$ et déposé dans les zones D1, D2 et D3 (Fig. 2) pendant le TRESBP

\begin{tabular}{|c|c|c|c|c|}
\hline Année & D1 & D2 & D3 & Total \\
\hline $\mathbf{1 9 9 5}$ & $?$ & $?$ & $?$ & 1731949 \\
\hline $\mathbf{1 9 9 6}$ & $?$ & $?$ & $?$ & 572403 \\
\hline $\mathbf{1 9 9 7 / 1 9 9 8}$ & $?$ & $?$ & $?$ & 800407 \\
\hline $\mathbf{2 0 0 0}$ & 237266 & 73010 & 96007 & 405853 \\
\hline $\mathbf{2 0 0 1}$ & 9721 & 257966 & 148490 & 416207 \\
\hline $\mathbf{2 0 0 2}$ & 190285 & 9014 & 40831 & 240130 \\
\hline $\mathbf{2 0 0 3}$ & 146628 & 57425 & 26839 & 230892 \\
\hline $\mathbf{2 0 0 4}$ & 123771 & 11200 & 34955 & 169926 \\
\hline $\mathbf{2 0 0 5}$ & 111483 & 38573 & 26133 & 176189 \\
\hline
\end{tabular}

Tableau 2. Quantités de sable pompé $\left(\mathrm{m}^{3}\right)$ aux différents émissaires indiqués sur la Figure 1 pendant le TRESBP

\begin{tabular}{|c|c|c|c|c|c|c|}
\hline Année & ESRE & ESRO & EK & ED & EG & Total \\
\hline $\mathbf{2 0 0 1}$ & 409746 & 1586 & 97279 & 67258 & 0 & 575869 \\
\hline $\mathbf{2 0 0 2}$ & 546577 & 0 & 84915 & 71872 & 0 & 721364 \\
\hline $\mathbf{2 0 0 3}$ & 602953 & 0 & 81918 & 70224 & 31931 & 787026 \\
\hline $\mathbf{2 0 0 4}$ & 459554 & 0 & 0 & 36813 & 0 & 496367 \\
\hline $\mathbf{2 0 0 5}$ & 683 & 0 & 0 & 41687 & 0 & 724931 \\
\hline
\end{tabular}

\subsection{Imagerie vidéo}

Depuis mi-avril 2002, un réseau de stations ARGUS (HOLMAN \& STANLEY, 2007) opère en continue en couvrant l'ensemble de la baie de Coolangatta avec un total de 16 caméras. La méthode PIC («Pixel Intensity Clustering », AARNINKHOF et al., 2003) est ici utilisée afin de déterminer chaque semaine la position du trait de côte au niveau des différents profils indiqués sur la Figure 2.

\section{$4 \quad$ Résultats}

\subsection{Evolution basse-fréquence ( année) de la baie de Coolangatta}

La Figure 3 montre l'évolution de la position du trait de côte depuis 1987 au niveau des 8 profils de référence. Les résultats montrent une première forte accrétion en 1990 qui est le résultat d'un placement de $3200000 \mathrm{~m}^{3}$ de sable directement sur le haut de plage (projet précédant le TRESBP qui n'est pas présenté dans ce papier). Une deuxième évolution forte correspond en 1995 à la première phase du TRESBP avec un placement de plus de $1700000 \mathrm{~m}^{3}$ (Tab. 1). 
L'évolution la plus significative dans l'ensemble de la baie est observée à partir de courant 2001 avec une forte avancée du trait de côte pouvant atteindre $200 \mathrm{~m}$ à Kirra Beach (profil K19, Fig. 3). Cette intense évolution coïncide avec la mise en place du système de bypass. La Figure 4 montre l'évolution de la morphologie de la baie de Coolangatta pendant le TRESBP entre 1997 et 2005. La forte accrétion de la baie est particulièrement visible encore une fois à partir de 2001. Cette sédimentation se caractérise dans un premier temps par une forte avancée de la plage à l'est de la baie au niveau de Rainbow Bay, puis courant 2002 dans Coolangatta Beach, et rapidement à Kirra Beach. Jusqu'à 2005, Coolangatta Beach et Rainbow Bay restent relativement stables tandis que Kirra Beach continue de sédimenter. Cette avancée du trait de côte est associée à la formation d'une barre pré-littorale quasi-rectiligne et à une forte remontée du niveau de la plage qui atteint par endroits $6 \mathrm{~m}$ par rapport à sa situation en 1995.
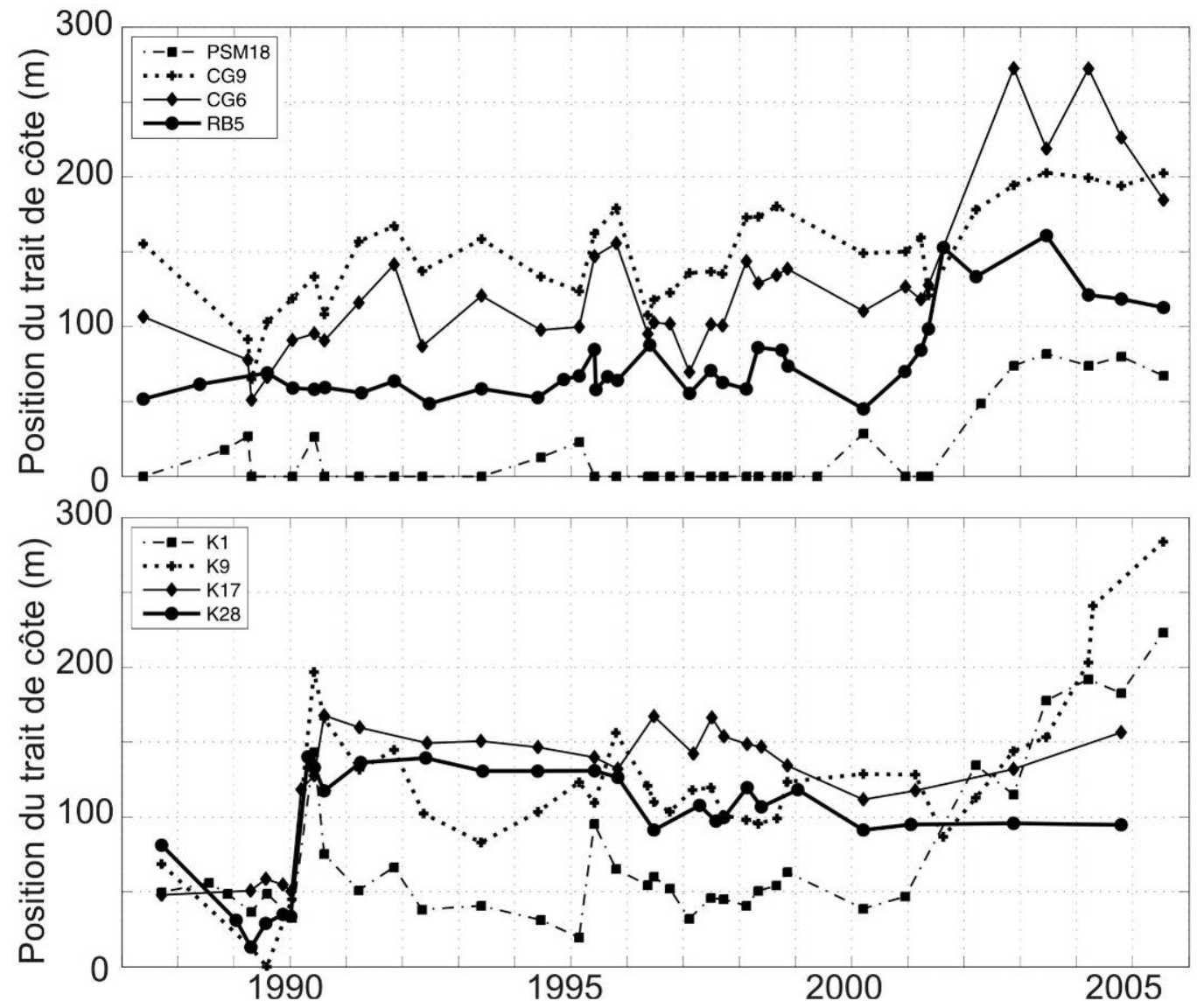

Figure 3. Evolution de du trait de côte au niveau des différents profils (Fig. 2) 

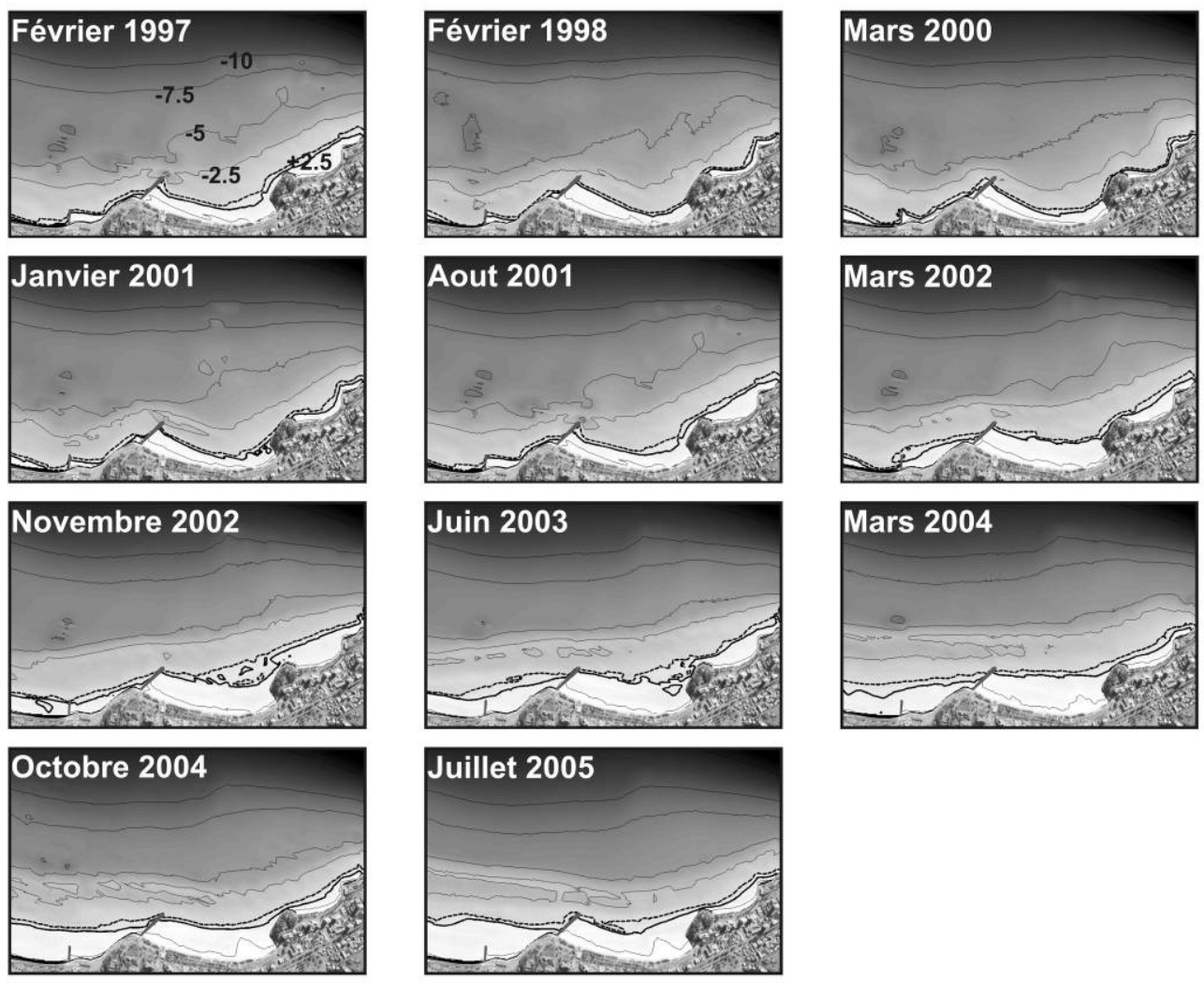

Figure 4. Evolution de la morphologie de la baie de Coolangatta à partir des relevés bathymétriques entre 1997 et 2005 pendant le TRESBP

\subsection{Evolution haute-fréquence ( $\sim$ semaine) de la baie de Coolangatta}

La Figure 5 montre l'évolution haute fréquence de la position du trait de côte déduite par imagerie vidéo ainsi que les quantités de sable surpompé modélisées entre 2002 et 2005. Les résultats montrent des variations haute-fréquence importantes facilement corrélables dans la partie est de la baie (RB5, CG6 et CG9) avec les quantités surpompées. Les calculs montrent que les fortes avancées sableuses formées par un surpompage significatif (par exemple début et fin 2003, Fig. 5) migrent à une vitesse moyenne de l'ordre de $100 \mathrm{~m} /$ mois vers l'ouest. Cette corrélation est particulièrement visible à Rainbow Bay située directement en aval de l'émissaire de Snapper Rock, alors qu'une avancée plus régulière est observée à Kirra Beach. Les résultats obtenus avec la modélisation numérique montrent que la méthode proposée par BERTIN et al. (2008) peut être appliquée pour mieux prévoir les quantités de sable à pomper en fonction des prévisions de houle. 

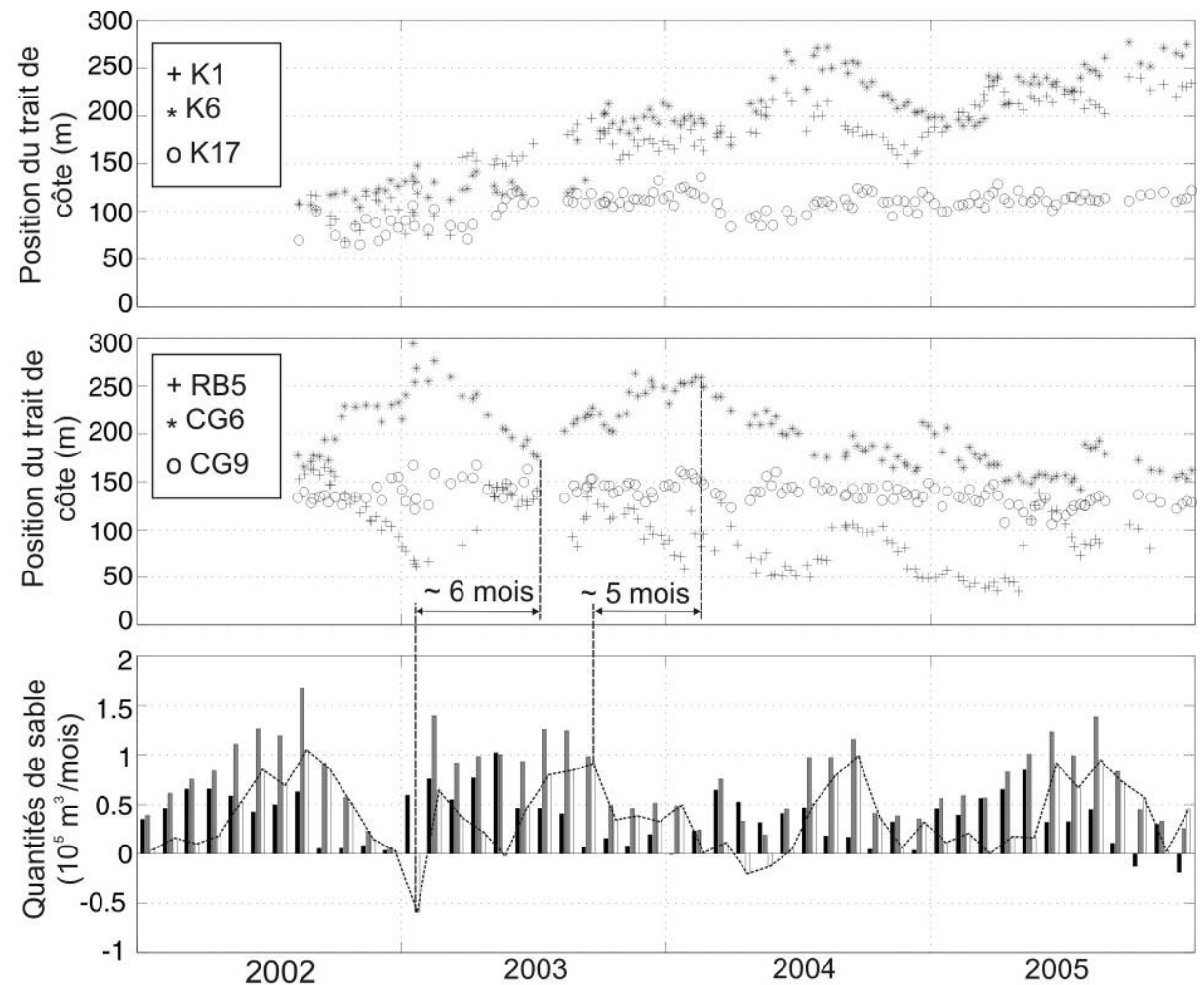

Figure 5. Evolution de la position du trait de côte déduite de l'imagerie vidéo et des quantités de sable surpompé (histogramme blanc) déduites de la modélisation

\section{$5 \quad$ Discussion et conclusions}

(1) Le système de bypass est bien plus performant que les rechargements réalisés avec les dépôts de dragage. En effet le sable pompé en eau peu profonde est immédiatement transporté par le courant de dérive et nourrit les bancs de sable puis le haut de plage de la baie.

(2) Les plages de la baie sont désormais très larges avec une avancée du trait de côte de 50 à $200 \mathrm{~m}$. Les plages de Coolangatta sont de nos jours les seules plages de la Gold Coast pouvant résister à une succession d'épisodes cycloniques tels que l'a connu la Gold Coast en 1967 (CASTELLE et al., 2008).

(3) Alors que Coolangatta Beach et Rainbow Bay se sont relativement stabilisées, Kirra Beach continue à sédimenter rapidement et une grande partie du sable pompé vient s'accumuler dans cette zone. Cela n'est pas complètement surprenant car Kirra Beach était la zone qui avait été la plus affectée par le piégeage de la dérive littoral par les aménagements de la Tweed River, et présentait donc le déficit sédimentaire le plus significatif.

(4) Cette rapide sédimentation de la baie est due aux quantités de sable surpompé pendant le TRESBP par rapport à dérive littorale naturelle. Cela est 
visible sur les échelles annuelles, mais aussi à plus haute fréquence où les avancées et reculs rapides du trait de côte peuvent être corrélés avec les quantités de sable surpompé calculées à partir de la modélisation numérique. Cette approche pourrait à l'avenir être utilisée afin de mieux gérer les quantités de sable pompé en fonction des prévisions de houle et ainsi reproduire au plus proche la dérive littorale naturelle.

(5) Il n'y a pas encore d'équilibre de la baie de Coolangatta. Il faudrait que les quantités de sable pompé se rapprochent des valeurs de la dérive littorale naturelle pendant quelques années afin que la morphologie de la baie devienne plus stable.

En comparaison avec l'état catastrophique des plages il y a 10 ans, le TRESBP a réussi avec succès à engraisser les plages de la baie et améliorer leur capacité à supporter les événements extrêmes. Par conséquent, les composantes ingénierie du projet sont un véritable succès, avec en particulier la mise en évidence de l'efficacité des systèmes de bypass. Ce projet a pourtant été, et est encore, très controversé. En effet, les plages sont désormais trop larges au goût de la communauté locale, en particulier Kirra Beach qui est abandonnée par les touristes. Les fortes accrétions observées dans la baie ont également recouvert une grande partie des récifs naturels immergés (voir Fig. 1), ce qui a conduit à la raréfaction de la faune et de la flore et donc à la rage des plongeurs et pêcheurs. Cet ensablement a également fait disparaitre la vague de Kirra qui était reconnue internationalement comme la meilleure vague au monde pour le surf.

Cette étude montre encore une fois la difficulté pour les projets de protection côtière à satisfaire à la fois la communauté et le cahier des charges étant donnée l'incompatibilité fréquente entre les différentes attentes.

\section{$6 \quad$ Remerciements}

Ces recherches ont été réalisées dans le cadre du Gold Coast Shoreline Management Plan financé par le Gold Coast City Council (GCCC). Les auteurs remercient le TRESBP pour avoir fourni une partie des relevés bathymétriques. Le financement permettant à la University of New South Wales d'opérer les stations ARGUS dans la baie de Coolangatta est apporté par le GCCC.

\section{$7 \quad$ Références bibliographiques}

1 AARNINKHOF S. G. J., TURNER I. L., DRONKERS T. D. T., CALJOUW M., NIPIUS L., (2003). A video-based technique for mapping intertidal beach bathymetry. Coastal Engineering 49 (4), pp 275-289.

2 BERTIN X., CASTELlE B., CHAUMILLON E., BUTEL R., QUIQUE R. (2008). Longshore transport estimation and inter-annual variability at a high- 
energy dissipative beach: St. Trojan beach, SW Oléron Island, France. Continental Shelf Research, 28, pp 1316-1332.

3 BOAK E. H., TURNER I. L., (2005). Shoreline definition and detection: A review. Journal of Coastal Research 21 (4), pp 688-703.

4 BOOIJ N., RIS R. C., HOLTHUIJSEN L. H., (1999). A third-generation wave model for coastal regions, Part I: Model description and validation. Journal of Geophysical Research 104 (C4), pp 7649-7666.

5 BROWNE M., STRAUSS D., CASTELLE B., BLUMENSTEIN M., TOMLINSON R. B., LANE C., (2006). Empirical estimation of nearshore waves from a global deep-water wave model. IEEE Geoscience and Remote Sensing Letters 3 (4), pp 462-466.

6 BROWNE M., CASTELlE B., STRAUSS D., TOMLINSON R. B., BLUMENSTEIN M., LANE C., (2007). Estimating onshore break size from a global wind-wave model: an empirical approach using artificial neural networks. Coast. Eng. 54 (5), pp 445-460.

7 CASTElle B., LE CORRE Y., TOMLINSON R.B. (2008). Can the Gold Coast beaches manage extreme events? Geo-Marine Letters 28, pp 23-30

8 CHARLIER R. H., CHAINEUX M. C. P., MORCOS S., (2005). Panorama of the history of coastal protection. Journal of Coastal Research 21 (1), pp 79-111.

9 HAMM L., CAPOBIANCO M., DETTE H. H., LECHUGA A., SPANHOff R., STIVE M. J. F., (2002). A summary of European experience with shore nourishment. Coastal Engineering 47, pp 237-264.

10 HANSON H., BRAMPTON A., CAPOBIANCO M., DETTE H.H., HAMM L., LAUSTRUP C., LECHUGA A., SPANHOff R., (2002). Beach nourishment projects, pratices, and objectives - a European overview. Coastal Engineering 47, pp 81-111.

11 HOLMAN R. A., STANLEY J., (2007). The history and technical capabilities of argus. Coastal Engineering 54 (6-7), pp 477-491

12 KACZMAREK L.M., OSTROWSKI R., PRUSZAK Z., ROZYNSKI G., (2005). Selected problems of sediment transport and morphodynamics of a multibar nearshore zone. Estuarine, Coastal and Shelf Science 62, pp 415-425.

13 TOLMAN H. L., (1991). A third-generation model for wind waves on slowly varying, unsteady and inhomogeneous depths and currents. Journal of Physical Oceanography 21, pp 782-797.

14 TURNER I. L., AARNINKHOF S. G. J., HOLMAN R. A., (2006). Coastal imaging applications and research in Australia. Journal of Coastal Research 22 (1), pp 37-48. 